\title{
CCL19 has potential to be a prognostic biomarker and a modulator of tumor immune microenvironment (TIME) of breast cancer: a comprehensive analysis based on TCGA database
}

\section{Jinyan Wang}

The Affiliated Jiangning Hospital of Nanjing Medical University

Jinqiu Wang

Dafeng People's Hospital

\section{Quan Gu}

Nanjing Medical University

\section{Yan Yang}

The Affiliated Jiangning Hospital

\section{Yajun Ma}

The Affiliated Jiangning Hospital of Nanjing Medical University

Quan'an Zhang ( $\nabla$ quananzhang_doctor@163.com )

The affiliated Jiangning Hospital of Nanjing Medical University https://orcid.org/0000-0002-63567122

\section{Research article}

Keywords: tumor microenvironment (TME), tumor immune microenvironment (TIME), tumor-infiltrating immune cells (TICs), CCL19, breast cancer

Posted Date: October 9th, 2020

DOI: https://doi.org/10.21203/rs.3.rs-87457/v1

License: (c) (i) This work is licensed under a Creative Commons Attribution 4.0 International License. Read Full License 


\section{Abstract}

The development of cancer was determined by not only the intrinsic properties of cancer cells, but also the communication between cancer cells and tumor microenvironment (TME). We applied ESTIMATE and CIBERSORT algorithms to calculate the immune/stromal component and tumor-infiltrating immune cells (TICs) in TME of BC. The results showed that immune component in TME predicted patients' survival and associated with progression of BC. Differentially expressed genes (DEGs) were primarily enriched in immune-related activities. Finally, CCL19 was acquired which shared the leading nodes in PPI network and was associated with patients' survival. High expression of CCL19 predicted better prognosis and participated in progression of BC. Genes in CCL19 up-regulated group were enriched in immune-related activities and these functions might depend on the communications between CCL19 and multiple TICs in TIME. In conclusion, CCL19 functioned as a potential prognostic biomarker and a modulator of TIME in $\mathrm{BC}$ through communicating with various TICs.

\section{Introduction}

Breast cancer (BC) is the most common cancer in women across the world, with an estimated 42,000 cancer-related deaths in the United States in 2020, and is the second leading cause of cancer-associated mortality ${ }^{[1]}$. Though, cytotoxic chemotherapy, including paclitaxel and carboplatin, has been widely used in the treatment of $B C$, the outcome of $B C$ patients is not yet satisfying, especially for patients with triplenegative breast cancer (TNBC) ${ }^{[2]}$. Therefore, it is urgent to further explore the underlying mechanisms of carcinogenesis and therapeutics in BC.

Accumulating evidence has demonstrated that the tumor microenvironment (TME) played a significant role in the occurrence and progression of $\mathrm{BC}{ }^{[3]}$. TME is made up of various cell types, such as fibroblasts, adipocyte, immune cells, inflammatory cells and blood and lymphatic vascular networks ${ }^{[4]}$. The communications between tumor cells and the surrounding TME obviously affect the genesis, progression and drug resistance of $\mathrm{BC}{ }^{[5]}$. Recently, the concept of the tumor immune microenvironment (TIME) has attracted increasing interests, which is composed of tumor-infiltrating immune cells (TICs), including natural killer (NK) cells, T cells (CD8,CD4), Treg, dentric cells (DCs), myeloid-derived suppressor cells (MDSCs), tumor-associated macrophages (TAMs) and so on ${ }^{[6]}$. Additionally, tumor cells exhibit the ability to inhibit the TIME and evade immune surveillance ${ }^{[7]}$. Meanwhile, TICs or immune-related genes in TIME act to predict the prognosis and therapeutic efficacy of cancer patients ${ }^{[8-10]}$. For instance, BC was previously recognized as a non-immunogenic tumor. Whereas, with the deepening of researches, it was found that tumor-infiltrating lymphocytes (TILs) were significantly associated with better prognosis in patients with early stage triple-negative and HER2-positive $B{ }^{[11]}$. This relevance brought light to the immune-based therapeutics, and led to the application of immune checkpoint blockers for BC patients. Therefore, it is meaningful to deeply understand the correlation between TIME and prognosis of $B C$, and identify the novel biomarkers and potential precise therapeutic targets of BC. 
Luckily, transcriptome profiling based on functional genomics analysis has shed light on the role of TME in BC. In our study, we assessed the ratio of immune/stromal component and the TIC proportion in TME of BC samples from The Cancer Genome Atlas (TCGA) database by using ESTIMATE and CIBERSORT algorithms. And then, we embarked from the differentially expressed genes (DEGs) obtained by comparison between immune component and stromal component in BC samples and clarified that C-C motif chemokine 19 (CCL19) functioned as a potential prognostic biomarker and a modulator of TIME in $\mathrm{BC}$ through communicating with various TICs in TIME.

\section{Methods}

\section{Data}

Transcriptome profiling and the corresponding clinical materials of BC samples and normal breast samples were downloaded from TCGA database (https://portal.gdc.cancer.gov/). According to the statistics, there were 1109 tumor samples and 113 normal samples in total.

\section{The calculation of Immune Score, Stromal Score, and ESTIMATE Score}

The ratio of immune or stromal component in TME of each tumor sample was analyzed in R language (version 3.6.3) with the help of ESTIMATE algorithm. Immune Score represented the proportion of immune ingredient in TME. Stromal Score represented the proportion of stromal ingredient in TME. ESTIMATE Score represented both the immune and stromal ingredient in TME.

\section{Survival Analysis}

Survival analysis was carried out with the help of survival and survminer packages in R. Kaplan-Meier plot and log-rank tests were performed to assess the associations between Immune/Stromal/ESTIMATE Score and the survival rate of BC patients. Survival analysis was also conducted to evaluate the relationship between the expression of CCL19 and the survival rate of BC patients. Univariate COX regression analysis was carried out by package survival in $\mathrm{R}$ to assess the influence of CCL19 on the survival of $B C$ patients. $P<0.05$ was considered to be statistically significant.

\section{Differential Analysis Between Immune/stromal/estimate Score And Clinic-pathological Features}

The differential analysis was carried out to evaluate the relationships between Immune/Stromal/ESTIMATE Score and clinic-pathological features, including stages and TNM classification. The differential analysis was also conducted to assess the association between the expression of CCL19 and clinic-pathological features. Wilcoxon rank sum and Kruskal-Wallis rank sum test were used for comparison.

\section{Confirmation Of Degs Regarding Immune Or Stromal Score}

1109 tumor samples were divided into the high-score group and the low-score group aompared with the median and based on the above calculation of Immune Score and Stromal Score. Package limma in R 
was used for data analysis. A fold change (FC, log2 (high-score/low-score) $)>1$ and false discovery rate $(F D R)<0.05$ were set up to search the DEGs. Heatmaps and venn diagram of DEGs were performed by using pheatmap package and VennDiagram package in $\mathrm{R}$, respectively.

\section{Gene ontology (GO) and Kyoto Encyclopedia of Genes and Genomes (KEGG) enrichment analysis}

ClusterProfiler, enrichplot, and ggplot2 packages in R were used for the GO and KEGG enrichment analysis of 223 DEGs. $P<0.05$ was considered to be statistically significant.

\section{Protein-Protein Interaction (PPI) Network and Gene Set Enrichment Analysis (GSEA)}

PPI network of 223 DEGs was downloaded from the Search Tool for Retrieval of Interacting Genes/Proteins (STRING) database (version 11.0) and further reconstructed in Cytoscape (3.6.1). The confidence of interactive relationship of nodes was greater than 0.95. GSEA (4.1.0) was used to evaluate the functional profile of CCL19. P $<0.05$ was considered to be statistically significant.

\section{Results}

\section{Analysis Procedure}

Firstly, we downloaded the transcriptome profiling and corresponding clinical materials of 1109 breast tumor samples and 113 normal breast samples from the TCGA database. Secondly, we evaluated the proportion of immune/stromal component in TME using ESTIMATE algorithm and the relationship between Immune/Stromal Score and survival rates or clinic-pathological characteristics of BC patients. Thirdly, a total of 223 DEGs were obtained based on the immune score and stromal score. GO and KEGG enrichment analysis and PPI network were performed to learn the specific biological functions of these DEGs. Univariate COX regression was used to assess the effects of these DEGs on BC patients' survival. Finally, CCL19 came to our eyes. And we focused on the association between the expression of CCL19 and the survival or clinic-pathological characteristics of BC patients. In addition, GSEA was carried out to find the biological functions of CCL19. CIBERSORT algorithm was applied to evaluate the TIC profile and correlation analysis was used to evaluate the relationship between the expression of CCL19 and the proportion of TICs in TIME.

\section{Immune component in TME was associated the survival of BC patients}

In order to explore the underlying relationship between the ratio of immune or stromal component in TME and the survival rate of $\mathrm{BC}$ patients, we first established the immune/stromal component evaluation system. The higher score reflected the larger amount of the immune or stromal component in TME. And then survival analysis was carried out by Kaplan-Meier plot and log-rank tests. The results showed that Immune Score was significantly associated with the survival rate of BC patients (Fig. $2 b ; p=0.008$ ), which meant that the higher Immune Score predicted the better survival. Yet, neither Stromal nor ESTIMATE Score was significantly related with the survival rate of BC patients (Fig. 2a, $c ; p=0.687$, 
0.339). The above results implied that the immune component in TME might be more suitable for indicating the prognosis of $\mathrm{BC}$ patients.

\section{Both immune and stromal component were significantly associated with the specific clinic- pathological features of $\mathrm{BC}$ patients}

To further determine the associations between scores and clinic-pathological features of BC patients, we analyzed the corresponding clinical materials (Supplement Table 1) of BC patients, including the age, stages and TNM classification. First, we found that Stromal, Immune and ESTIMATE Score were significantly associated with the age of $\mathrm{BC}$ patients, which might indicate that the component of TME changed with age (Fig. 2d, i, n; $p=0.014,0.03,0.0068$ ). For other clinic-pathological features, we found that there was significant difference in the Stormal Score in stage I compared with stage II (Fig. 2e, $p=$ 0.017). There was also significant difference in the Stormal Score in stage T1 compared with stage T2 or T3, in stage N0 compared with stage N2 (Fig. 2f-g, $p=0.00023,0.022,0.015$ ). However, for Immune Score, we were unable to find any difference in stages or TNM classifications (Fig. 2j-m). In addition, for ESTIMATE Score, there was also significant difference between T1 and T2 or T4 (Fig. 2p, $p=0.016$, 0.029). Nonethless, none of Immune, Stromal or ESTIMATE Score was related with the distant metastasis of $B C$ (Fig. $2 h, m, f ; p>0.05$ ). From above, we concluded that the proportion of immune and stromal component might be associated with the early progression of $\mathrm{BC}$, instead of the distant metastasis.

\section{DEGs Were Significantly Related With Immune-related Activities}

In order to obtain the DEGs profile in TME, we performed the difference analysis between the high Stromal/Immune Score group and the low Stromal/Immune Score group. Heat map was ploted to show the gene profile in TME (Fig. 3a-b). In detail, there were a total of 773 DEGs obtained from the Stromal Score group (high Stromal Score vs. low Stromal Score), among which 634 DEGs were up-regulated and 139 DEGs were down-regulated (Fig. 3a, Supplement Table 2). Additionally, there were a total of 832 DEGs obtained from the Immune Score group (high Immue Score vs. low Immue Score), among which 729 DEGs were up-regulated and 103 DEGs were down-regulated (Fig. 3b, Supplement Table 3). Next, we conducted the intersection analysis to acquire the commonly up-regulated or down-regulated DEGs in Immune and Stromal Score groups. The venn diagram showed that 193 DEGs were up-regulated and 30 DEGs were down-regulated in both Immune Score group and Stromal Score group (Fig. 3c). Thus, these 223 DEGs (Supplement Table 4) might take a great part in modulating the status of TME. As a result, GO and KEGG enrichment analyses were further performed to assess the detailed functions of these 223 DEGs. Go enrichment analysis indicated that 223 DEGs greatly participated in lymphocyte activation, $T$ cell activation and T cell differentiation (Fig. 3d). Similarly, KEGG enrichment analysis revealed that 223 DEGs were significantly associated with specific immune-related activities, such as cell adhesion molecules, cytokine-cytokine receptor interaction and chemokine signaling pathway (Fig. 3e-g). From above, these 223 DEGs seemed to be closely related with immune-related activities and might be a primary feature of TME in BC.

\section{PPI network and univariate COX regression analysis of DEGs}


To learn more about the potential interaction mechanisms of these 223 DEGs, PPI network was first constructed with the help of STRING database and Cytoscape software. The specific interaction network of DEGs was showed in Fig. 4a. The top 10 DEGs, which shared the leading nodes, were ranked in Fig. 4b. We next conducted the univariate COX regression analysis to acquire the DEGs, which were significantly associated with $\mathrm{BC}$ patients' survival. As a result, $32 \mathrm{DEG}$ were found to be closely related with the hazard ratio (HR) of BC patients' survival (Fig. 4c, p< 0.05; Supplement Table 5). Finally, the intersection analysis was used to find out the DEGs, which shared the leading nodes in PPI and were significantly associated with patients' survival (Fig. 4d). Luckily, C-C motif chemokine 19 (CCL19) emerged.

\section{CCL19 was significantly associated with the survival and specific clinic-pathological features of BC patients}

CCL19, a ligand of the chemokine receptor C-C chemokine receptor type 7 (CCR7), is often expressed in the T-cell zones, including lymph nodes and thymus ${ }^{[12]}$. Recently, researches revealed that the

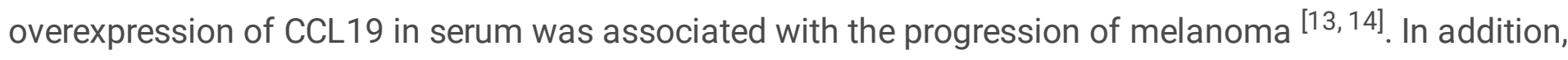
CCL19 functioned as an immune-modulator for colon cancer therapy by closely communicating with DCs, $T$ cells and $B$ cells, thus regulating the adaptive immune responses ${ }^{[15]}$. However, there are few researches focusing on the role of CCL19 in BC. In the present study, the survival analysis suggested that the expression of CCL19 was positively related with the survival of $B C$ patients (Fig. 5a, p =0.009). As for the clinic-pathological features, the expression of CCL19 was significantly associated with ages, stages, $\mathrm{T}$ classifications and $\mathrm{N}$ classifications of BC patients (Fig. $5 \mathrm{~b}-\mathrm{e}, \mathrm{p}<0.05$ ). However, it was not connected with $\mathrm{M}$ classification (Fig. 5f, $\mathrm{p}>0.05$ ), which was in accordance with the difference analysis between Score and clinic-pathological features (Fig. $2 h, m, f ; p>0.05$ ). From above, we might conclude that CCL19 was likely to be a prognostic factor and participate in the early progression of $\mathrm{BC}$.

\section{CCL19 Greatly Participated In Immune-related Activities}

Given that CCL19 was significantly associated with the survival and specific clinic-pathological features of BC patients, we further carried out GSEA to explore the underlying mechanisms of CCL19. First, BC samples were divided into two groups according to the expression of CCL19, including CCL19 upregulated group and CCL19 down-regulated group, compared with the median. For C2 collection, the KEGG gene sets database, genes in CCL19 up-regulated group were obviously enriched in immune-related activities, including $B$ cell receptor signaling pathway, cell adhesion molecules cams, chemokine signaling pathway and so on (Fig. 5g). For hallmark gene sets, genes in CCL19 up-regulated group were primarily enriched in allograft rejection, complement, IL2 stat5 signaling and so on (Fig. 5h). For C7 collection defined by MSigDB, the immunologic gene sets, a variety of immune functional gene sets were enriched in the high CCL19 expression group (Fig. 5i). At last, for C5 collection, the gene ontology sets, the genes in CCL19 up-regulated group were also enriched in immune-related activities, such as adaptive immune response, cytokine binding, cytokine receptor activity and so on (Fig. 5j). Thus, we concluded that CCL19 greatly participated in the immune-related activities in BC patients and might be a modulator of TME. 


\section{CCL19 Was Associated With Tics In Time}

Since we had found that CCL19 greatly participated in immune-related activities and might play an important role in the regulation of TME, we speculated that there were underlying connections between CCL19 and TIME. CIBERSORT algorithm was first applied to evaluate the proportion of TICs in BC samples (Fig. 6a). The associations between TICs were displayed in Fig. 6b. The difference analysis was performed to verify the associations between the expression of CCL19 and 22 TICs. The violin plot suggested that 15 TICs were significantly associated with the expression of CCL19 in BC samples (Fig. 6c, Supplement Table 6). Next, the correlation test showed that 10 TICs were positively related with the expression of CCL19 (Fig. 7a-j, Supplement Table 6), and 6 TICs were negatively related with the expression of CCL19 (Fig. 7k-p, Supplement Table 6). Finally, the intersection analysis between the difference test and correlation test revealed that 15 TICs were significantly associated with the expression level of CCL19 (Fig. 6d, Supplement Table 6). As a consequence, CCL19 was significantly associated with multiple TICs in TIME and might play an important role in modulating the TIME of BC through communicating with various TICs.

\section{Discussion}

In our study, we focused on the TME-related genes that were significantly associated with the survival and the clinic-pathological features of BC. Firstly, we downloaded the transcriptome profiling and the clinical data of 1109 breast tumor samples and 113 normal breast samples from the TCGA database. Secondly, we applied the ESTIMATE algorithm to calculate the ratio of immune/stromal component in TME and found out that immune/stromal component in TME was closely related with the survival rate and clinic-pathological characteristics of BC patients. Thirdly, in order to further explore the gene expressing profiling regarding the TME, 223 DEGs were obtained based on the Immune Score and Stromal Score. GO and KEGG enrichment analysis indicated that a total of 223 DEGs were primarily enriched in immune-related activities, such as lymphocyte activation, $\mathrm{T}$ cell activation, $\mathrm{T}$ cell differentiation, cytokine-cytokine receptor interaction, chemokine signaling pathway and so on. Finally, CCL19 was acquired by intersection analysis between PPI network and univariate COX regression analysis. CCL19 shared the leading nodes in PPI network and was significantly associated with the HR of $B C$ patients' survival. And then survival analysis and differential analysis revealed that the overexpression of CCL19 predicted the better survival and clinic-pathological characteristics of BC patients. Additionally, GSEA revealed that genes in CCL19 up-regulated group were mainly enriched in immune-related activities, such as B cell receptor signaling pathway, chemokine signaling pathway, allograft rejection, cytokine receptor activity and so on. Furthermore, CCL19 was closely associated with a variety of TICs in TIME and might play an important role in modulating TIME through communicating with multiple TICs.

CCL19, a ligand of CCR7, was overexpressed in the T-cell zones and functioned as an essential modulator of immune responses through affecting the migration of DCs and T cells into the secondary lymphatic tissues and thereby influencing cell homing and adaptive immunity [16-20]. Recently, H. W. Cheng, et al. [21] declared that CCL19-expressing fibroblastic stromal cells (FSCs) accelerated the intratumoral 
accumulation of CD8 + T cells and inhibited the tumor growth in non-small cell lung cancer. CCL19, along with CCR7, acted as clinically prognostic factors in lung adenocarcinoma ${ }^{[22]}$. In addition, CCL19expressing chimeric antigen receptor (CAR) T cells promoted the infiltration of DCs and T cells into tumor tissues and exerted synergistic anti-tumor activity with recipient immune cells ${ }^{[23]}$. In other solid tumors, such as ovarian and colon cancers, CCL19-expressing embryonic endothelial progenitor cells and mesenchymal stem cells functioned to activate the local TIME and led to a good therapeutic effect $[24,25]$. On the other hand, immune cells, such as DCs, can also release CCL19 during the migration, to maintain immune surveillance and tissue homeostasis ${ }^{[26,27]}$. However, there are few researches exploring the role of CCL19 in BC and the correlation between the expression of CCL19 and other TICs. H. Hwang, et al. [28] indicated that TNBC cells advanced the migration of DCs toward CCL19 through releasing soluble factors and activating JNK/c-Jun signaling pathway.

In our study, we applied multiple analytical methods to evaluate the functions of DEGs regarding stromal and immune component in TME of BC samples from TCGA and finally found out the most significant gene, CCL19. Further analysis between the expression of CCL19 and the survival of patients or TICs in TIME suggested that CCL19 was not only significantly associated with the survival and clinicpathological characteristics of BC patients, but also participated in the regulation of TIME partly through closely communicating with multiple TICs, including macrophages, T cells, B cells, NK cells, DCs, mast cells and neutrophils. Previous studies indicated that CCL19 greatly participated in cancer cell proliferation, angiogenesis, migration, invasion and immune cell infiltration ${ }^{[23]}$ in multiple cancers, including gastric cancer ${ }^{[29]}$, colorectal cancer ${ }^{[30]}$, non-small cell lung cancer ${ }^{[31,32]}$, lymphoma ${ }^{[33]}$ and melanoma ${ }^{[13]}$. These biological functions of CCL19 were involved in the activation of CCR7/absent in melanoma 2 (AIM2) signaling pathway ${ }^{[29]}$, interaction with miRNAs ${ }^{[30]}$, the migration and maturation of DC ${ }^{[34]}$ and migration of NK cells ${ }^{[35]}$. Our studies provided a new point of view that CCL19 might not only be connected with the migration and maturation of DCs or NK cells, but also associated with macrophages, $T$ cells, B cells, mast cells and neutrophils. And further researches were needed to clarify the specific associations between CCL19 and multiple TICs in BC.

\section{Conclusion}

We applied ESTIMATE algorithm to assess the immune and stromal component in TME of BC samples downloaded from TCGA database and identified TME-associated DEGs through multiple analytical methods. The results indicated that CCL19 was a potential prognostic biomarker for BC patients. More interestingly, CCL19 might function as a modulator of BC TIME and is significantly connected with various TICs. Therefore, further investigation should be carried out to clarify the accuracy of the combined analysis of CCL19 expression, explore the role of CCL19 in TIME of BC and focus more on the correlation between CCL19 and these immune cells instead of just aiming at DCs.

\section{Declarations}




\section{Ethics approval and consent to participate}

Not applicable.

\section{Consent for publication}

Not applicable.

\section{Availability of data and material}

The datasets generated and analyzed in this research are available in TCGA database (https://portal.gdc.cancer.gov)

\section{Competing interests}

The authors declare that they have no competing interests.

\section{Fundings}

This research was funded by the Natural Science Foundation of Jiangsu Province, grant number BK20161110, the Priority Academic Program Development of Jiangsu Higher Education Institutions, grant number JX10231802, Postgraduate Research and Practice Innovation Program of Jiangsu Province, grant number SJCX17_0387, the Science Foundation of Jiangsu Health vocational college, grant number JKC201948, the Science and Technology Development Fund of Nanjing Medical University, grant number NMUB2019235, the Nanjing health science and Technology Development Fund, grant number YKK18201, Science and technology development fund of Nanjing Medical University, grant number 2017NJMUZD091 and the Research and development fund of Kangda College of Nanjing Medical University, grant number KD2017KYJJYB017.

Author Contributions $\rrbracket$ Jinyan Wang: conceptualization, formal analysis, funding acquisition, visualization, Writing - original draft; Jinqiu Wang: data curation, project administration, resources, Writing - original draft; Quan Gu: investigation, methodology, software, Writing - original draft; Yan Yang: funding acquisition; Yajun Ma: supervision; Quanan Zhang: funding acquisition, validation, Writing - review \& editing.

\section{References}

1. Goding Sauer A, Fedewa SA, Butterly LF, Anderson JC, Cercek A, Smith RA, et al. Cancer statistics, 2020. CA Cancer J Clin. 2020; 70: 7-30.

2. Lu H, Xie Y, Tran L, Lan J, Yang Y, Murugan NL, et al. Chemotherapy-induced S100A10 recruits KDM6A to facilitate OCT4-mediated breast cancer stemness. J Clin Invest. 2020; 130: 4607-23.

3. Wang J, Zhang Q, Wang D, Yang S, Zhou S, Xu H, et al. Microenvironment-induced TIMP2 loss by cancer-secreted exosomal miR-4443 promotes liver metastasis of breast cancer. 2020. 
4. Grivennikov SI, Greten FR, Karin M. Immunity, inflammation, and cancer. Cell. 2010; 140: 883-99.

5. Bareche Y, Buisseret L, Gruosso T, Girard E, Venet D, Dupont F, et al. Unraveling Triple-Negative Breast Cancer Tumor Microenvironment Heterogeneity: Towards an Optimized Treatment Approach. J Natl Cancer Inst. 2020; 112: 708-19.

6. Baba Y, Nomoto D, Okadome K, Ishimoto T. Tumor immune microenvironment and immune checkpoint inhibitors in esophageal squamous cell carcinoma. 2020; 111: 3132-41.

7. Gajewski TF, Schreiber H, Fu YX. Innate and adaptive immune cells in the tumor microenvironment. Nat Immunol. 2013; 14: 1014-22.

8. Zhang L, Chen J, Cheng T, Yang H, Li H, Pan C. Identification of the key genes and characterizations of Tumor Immune Microenvironment in Lung Adenocarcinoma (LUAD) and Lung Squamous Cell Carcinoma (LUSC). J Cancer. 2020; 11: 4965-79.

9. Yu H, Zhu X, Lin $\mathrm{H}$, Pan $\mathrm{H}$, Zhao F, Zhu M, et al. A new risk model comprising genes highly correlated with CD133 identifies different tumor-immune microenvironment subtypes impacting prognosis in hepatocellular carcinoma. J Cell Physiol. 2020; 12: 12234-50.

10. Zhao B, Wang Y, Wang Y, Chen W, Liu PH, Kong Z, et al. Systematic identification, development, and validation of prognostic biomarkers involving the tumor-immune microenvironment for glioblastoma. 2020.

11. Savas P, Salgado R, Denkert C, Sotiriou C, Darcy PK, Smyth MJ, et al. Clinical relevance of host immunity in breast cancer: from TILs to the clinic. Nat Rev Clin Oncol. 2016; 13: 228-41.

12. Yoshida R, Nagira M, Imai T, Baba M, Takagi S, Tabira Y, et al. EBI1-ligand chemokine (ELC) attracts a broad spectrum of lymphocytes: activated T cells strongly up-regulate CCR7 and efficiently migrate toward ELC. Int Immunol. 1998; 10: 901-10.

13. Cristiani CM, Turdo A, Ventura V, Apuzzo T, Capone M, Madonna G, et al. Accumulation of Circulating CCR7(+) Natural Killer Cells Marks Melanoma Evolution and Reveals a CCL19-Dependent Metastatic Pathway. Cancer Immunol Res. 2019; 7: 841-52.

14. Fujimura T, Tanita K, Sato Y, Lyu C, Kambayashi Y, Fujisawa Y, et al. Immune checkpoint inhibitorinduced vitiligo in advanced melanoma could be related to increased levels of CCL19. Br J Dermatol. 2020; 182: 1297-300.

15. Liu X, Wang B, Li Y, Hu Y, Li X, Yu T, et al. Powerful Anticolon Tumor Effect of Targeted Gene Immunotherapy Using Folate-Modified Nanoparticle Delivery of CCL19 To Activate the Immune System. ACS Cent Sci. 2019; 5: 277-89.

16. Radstake TR, van der Voort R, ten Brummelhuis M, de Waal Malefijt M, Looman M, Figdor CG, et al. Increased expression of CCL18, CCL19, and CCL17 by dendritic cells from patients with rheumatoid arthritis, and regulation by Fc gamma receptors. Ann Rheum Dis. 2005; 64: 359-67.

17. Viola A, Contento RL, Molon B. T cells and their partners: The chemokine dating agency. Trends Immunol. 2006; 27: 421-7.

18. Bromley SK, Mempel TR, Luster AD. Orchestrating the orchestrators: chemokines in control of T cell traffic. Nat Immunol. 2008; 9: 970-80. 
19. Buonamici S, Trimarchi T, Ruocco MG, Reavie L, Cathelin S, Mar BG, et al. CCR7 signalling as an essential regulator of CNS infiltration in T-cell leukaemia. Nature. 2009; 459: 1000-4.

20. Comerford I, Harata-Lee Y, Bunting MD, Gregor C, Kara EE, McColl SR. A myriad of functions and complex regulation of the CCR7/CCL19/CCL21 chemokine axis in the adaptive immune system. Cytokine Growth Factor Rev. 2013; 24: 269-83.

21. Cheng HW, Onder L, Cupovic J, Boesch M, Novkovic M, Pikor N, et al. CCL19-producing fibroblastic stromal cells restrain lung carcinoma growth by promoting local antitumor T-cell responses. J Allergy Clin Immunol. 2018; 142: 1257-71.e4.

22. Itakura M, Terashima Y, Shingyoji M, Yokoi S, Ohira M, Kageyama H, et al. High CC chemokine receptor 7 expression improves postoperative prognosis of lung adenocarcinoma patients. $\mathrm{Br} \mathrm{J}$ Cancer. 2013; 109: 1100-8.

23. Adachi K, Kano Y, Nagai T, Okuyama N, Sakoda Y, Tamada K. IL-7 and CCL19 expression in CAR-T cells improves immune cell infiltration and CAR-T cell survival in the tumor. Nat Biotechnol. 2018; 36: 346-51.

24. Hamanishi J, Mandai M, Matsumura N, Baba T, Yamaguchi K, Fujii S, et al. Activated local immunity by CC chemokine ligand 19-transduced embryonic endothelial progenitor cells suppresses metastasis of murine ovarian cancer. Stem Cells. 2010; 28: 164-73.

25. lida Y, Yoshikawa R, Murata A, Kotani H, Kazuki Y, Oshimura M, et al. Local injection of CCL19expressing mesenchymal stem cells augments the therapeutic efficacy of anti-PD-L1 antibody by promoting infiltration of immune cells. J Immunother Cancer. 2020; 8.

26. Worbs T, Hammerschmidt SI, Förster R. Dendritic cell migration in health and disease. Nat Rev Immunol. 2017; 17: 30-48.

27. Förster R, Davalos-Misslitz AC, Rot A. CCR7 and its ligands: balancing immunity and tolerance. Nat Rev Immunol. 2008; 8: 362-71.

28. Hwang H, Shin C, Park J, Kang E, Choi B, Han JA, et al. Human breast cancer-derived soluble factors facilitate CCL19-induced chemotaxis of human dendritic cells. Sci Rep. 2016; 6: 30207.

29. Zhou R, Sun J, He C, Huang C, Yu H. CCL19 suppresses gastric cancer cell proliferation, migration, and invasion through the CCL19/CCR7/AIM2 pathway. Hum Cell. 2020.

30. Xu Z, Zhu C, Chen C, Zong Y, Feng H, Liu D, et al. CCL19 suppresses angiogenesis through promoting miR-206 and inhibiting Met/ERK/Elk-1/HIF-1a/VEGF-A pathway in colorectal cancer. Cell Death Dis. 2018; 9: 974.

31. Baran K, Kiszałkiewicz J, Migdalska-Sęk M, Jabłoński S, Kordiak J, Antczak A, et al. An assessment of the relationship between the expression of CCR7/CCL19 axis and selected regulatory miRNAs in non-small cell lung cancer. Mol Biol Rep. 2019; 46: 5389-96.

32. Kim HJ, Park J, Lee SK, Kim KR, Park KK, Chung WY. Loss of RUNX3 expression promotes cancerassociated bone destruction by regulating CCL5, CCL19 and CXCL11 in non-small cell lung cancer. J Pathol. 2015; 237: 520-31. 
33. O'Connor T, Zhou X, Kosla J, Adili A, Garcia Beccaria M, Kotsiliti E, et al. Age-Related Gliosis Promotes Central Nervous System Lymphoma through CCL19-Mediated Tumor Cell Retention. Cancer Cell. 2019; 36: 250-67.e9.

34. Qian J, Xu X, Ding J, Yin R, Sun Y, Xue C, et al. Newcastle disease virus-like particles induce DC maturation through TLR4/NF-KB pathway and facilitate DC migration by CCR7-CCL19/CCL21 axis. Vet Microbiol. 2017; 203: 158-66.

35. Carlsten M, Levy E, Karambelkar A, Li L, Reger R, Berg M, et al. Efficient mRNA-Based Genetic Engineering of Human NK Cells with High-Affinity CD16 and CCR7 Augments Rituximab-Induced ADCC against Lymphoma and Targets NK Cell Migration toward the Lymph Node-Associated Chemokine CCL19. Front Immunol. 2016; 7: 105.

\section{Figures}

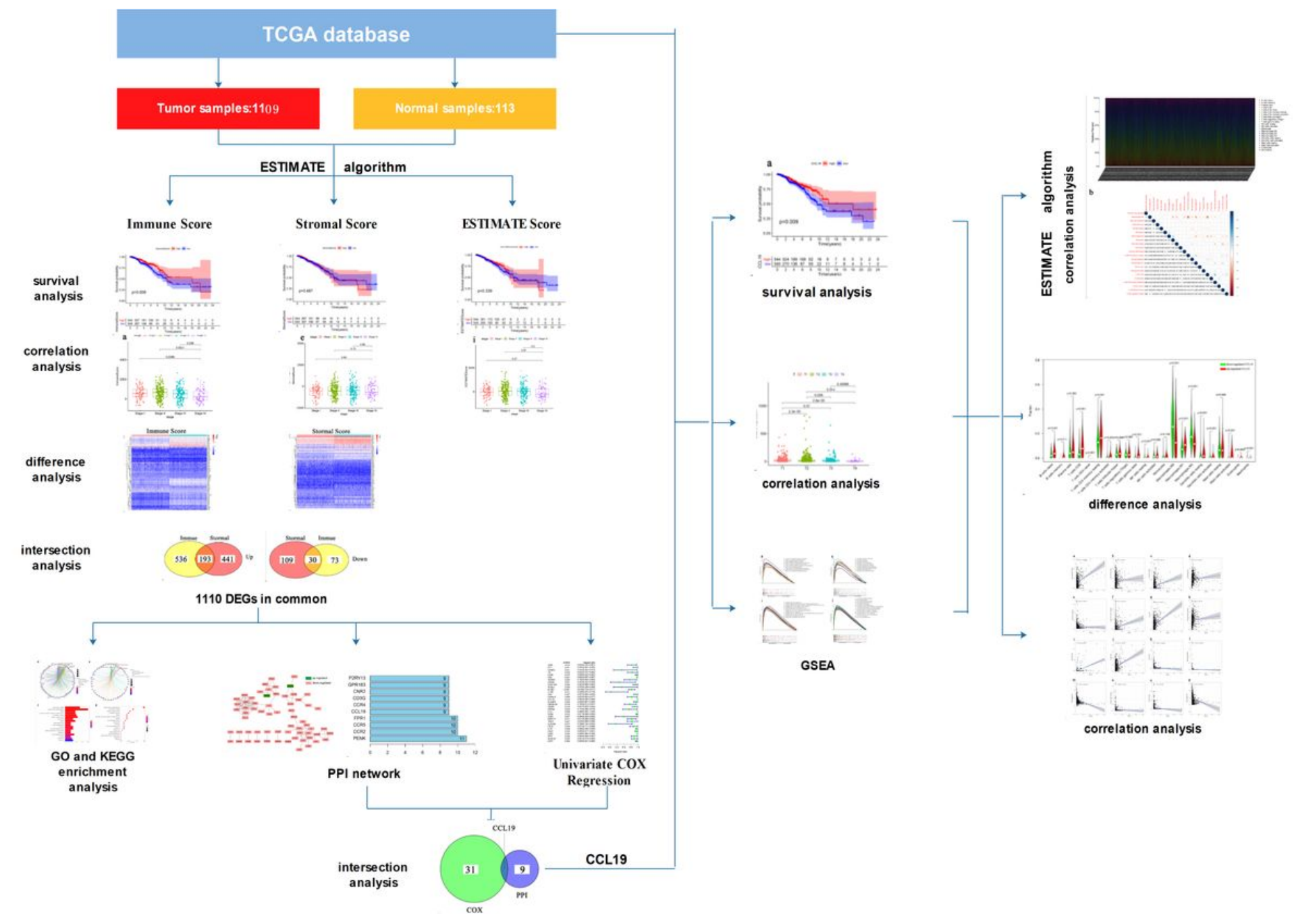

\section{Figure 1}

The analysis procedure of this study. 

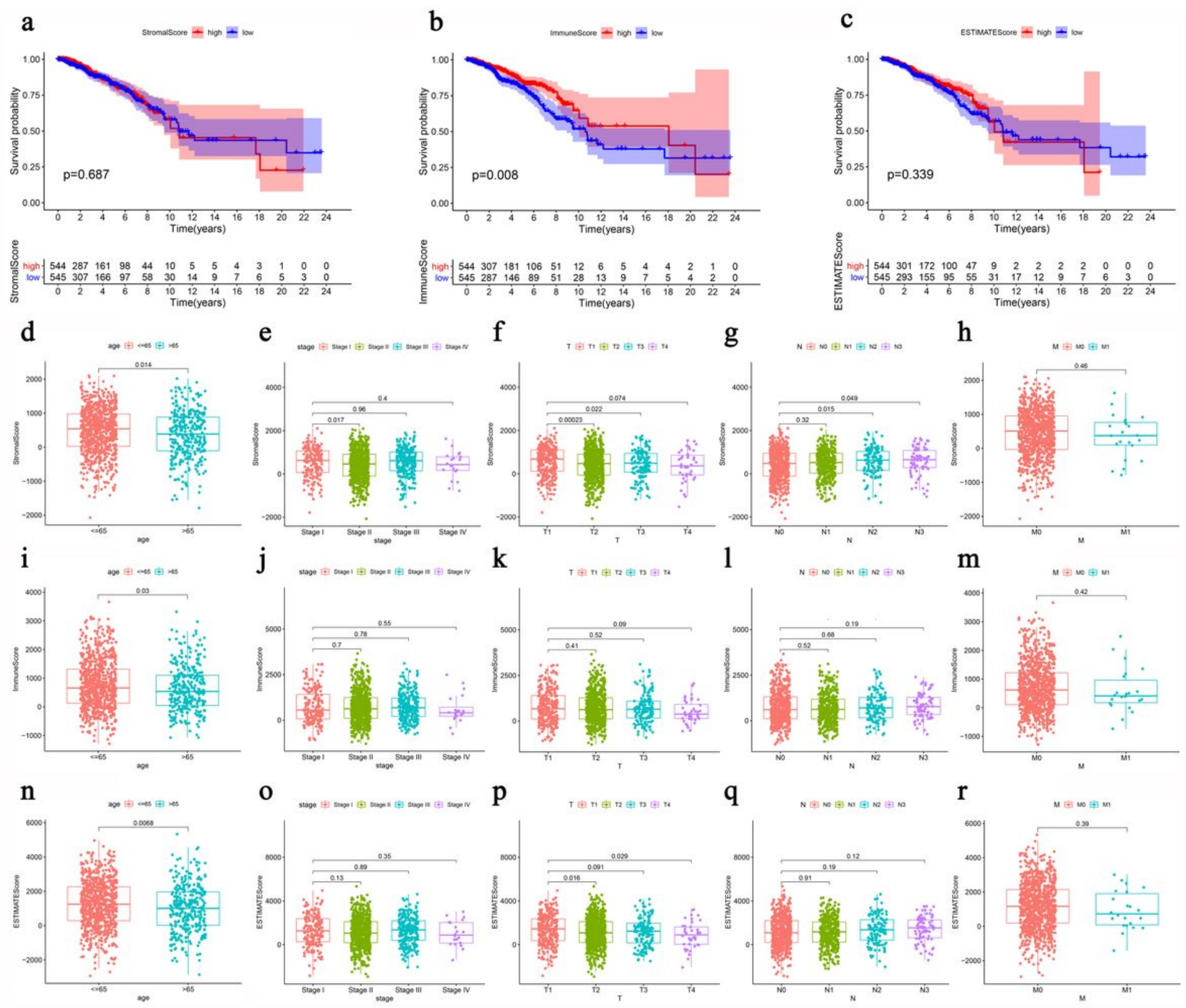

\section{Figure 2}

The association of Immune/Stromal/ESTIMATE Score and the survival or clinic-pathological characteristics of breast cancer patients. (a-c) Kaplan-Meier survival analysis was applied to evaluate the associations between Immune/Stromal/ESTIMATE Score and the survival of breast cancer patients. Patients were divided into two groups, high score group and low score group, compared with the median. P-value and the number of samples in each group were displayed in the diagram; (d-h) The associations of Stromal Score with age, stage and TNM classification of breast cancer patients; (i-m) The associations of Immune Score with age, stage and TNM classification of breast cancer patients; (n-r) The associations of ESTIMATE Score with age, stage and TNM classification of breast cancer patients. 
a

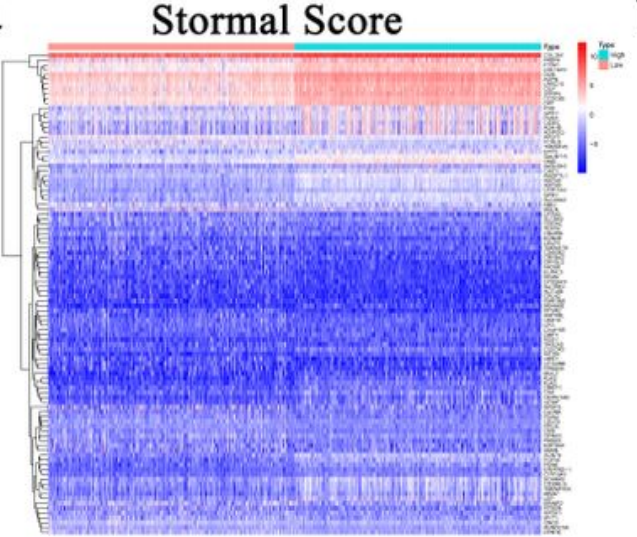

b

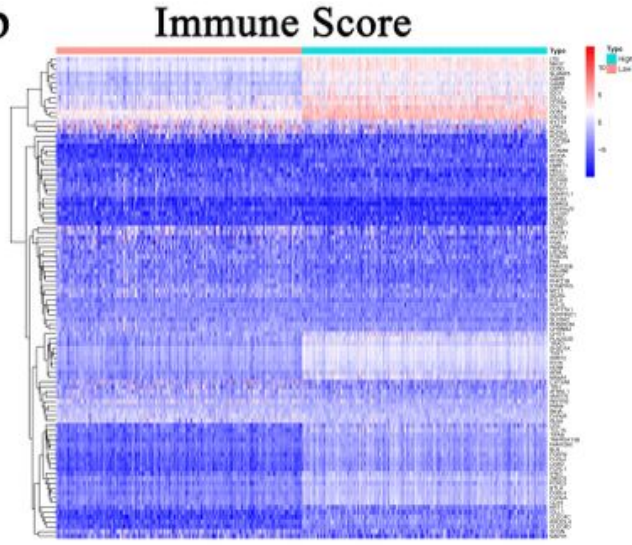

C
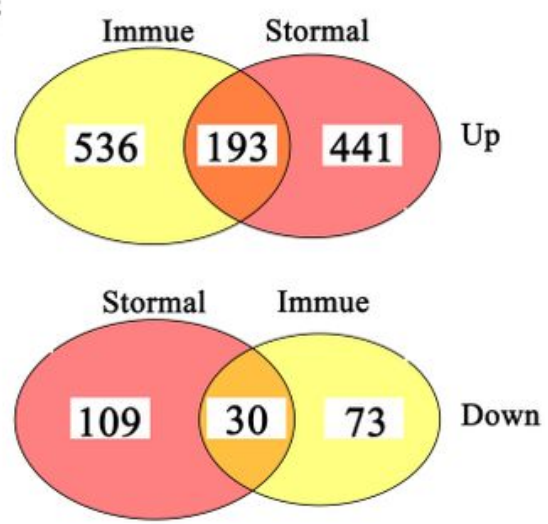

d

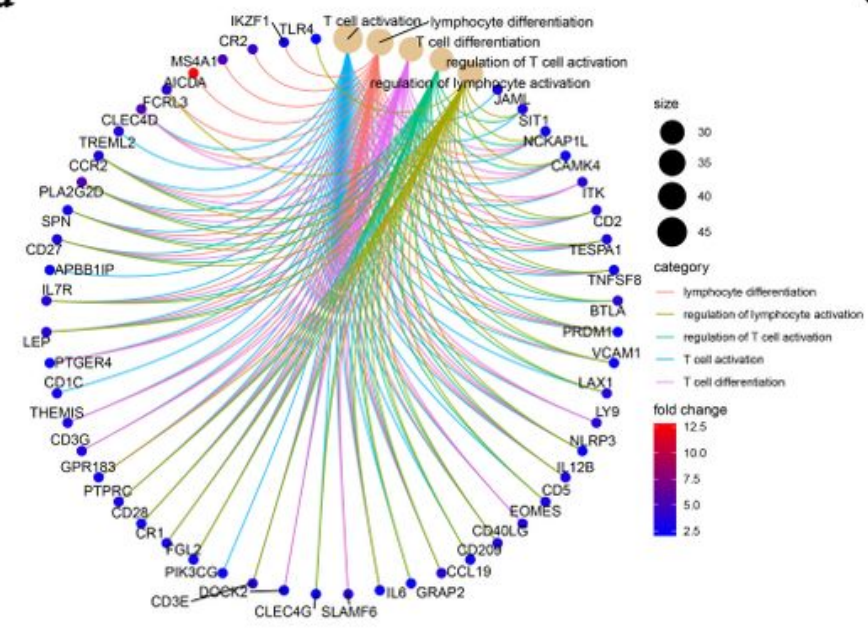

f

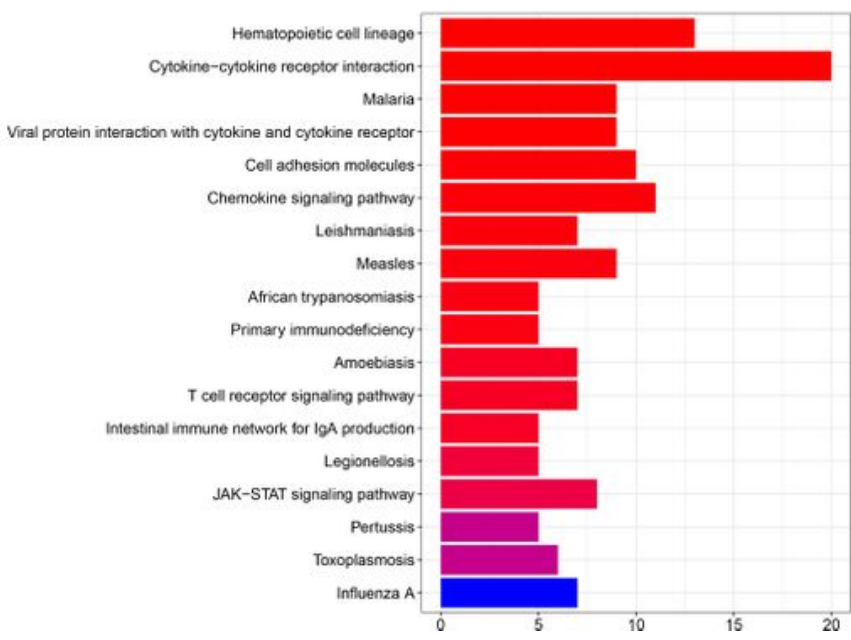

e

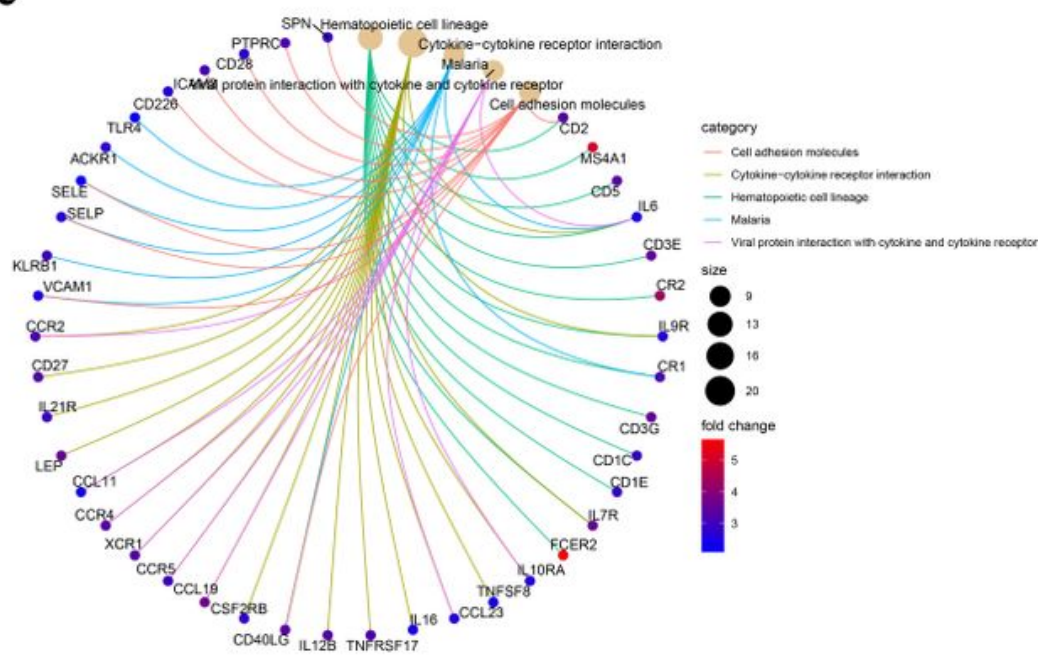

g

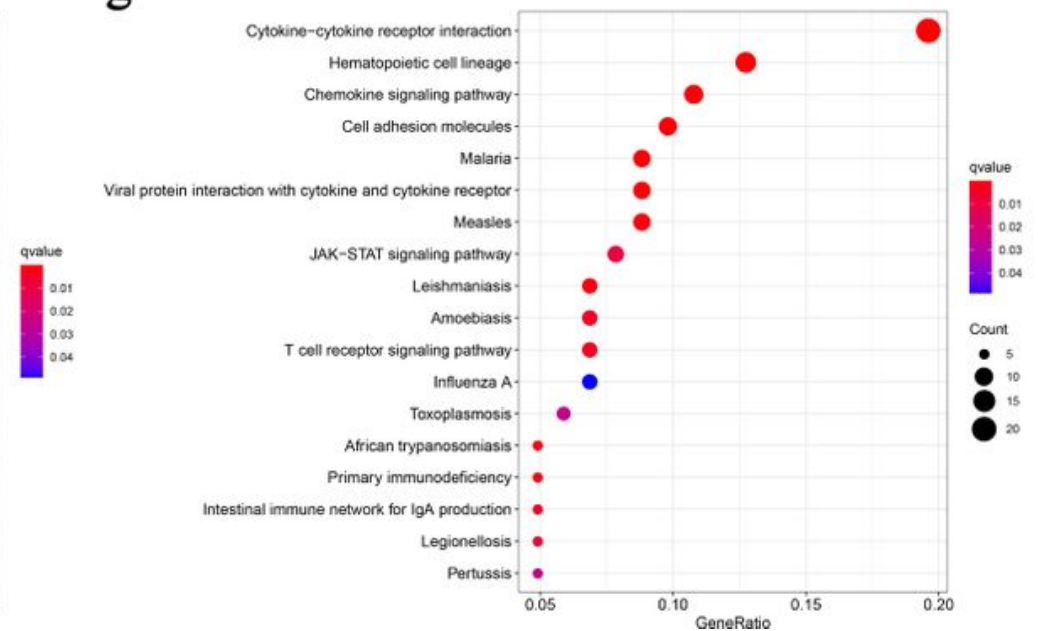

\section{Figure 3}

Heatmaps, venn plots, GO and KEGG enrichment analyses. (a-b) Heatmaps of DEGs obtained by comparison between high Stromal/Immune Score samples with low Stromal/Immune Score samples. $p<0.05$ and log2FC $>1$ were set up to search the DEGs; (c) Venn plots displayed the commonly upregulated or down-regulated DEGs shared by Immune Score and Stromal Score; (d) GO enrichment analysis of 223 DEGs, p<0.05 was considered to be statistically significant; (e-g) KEGG enrichment 
analysis of 223 DEGs, $p<0.05$ was considered to be statistically significant; BP: biological process; CC: cell component; MF: molecular function.

a

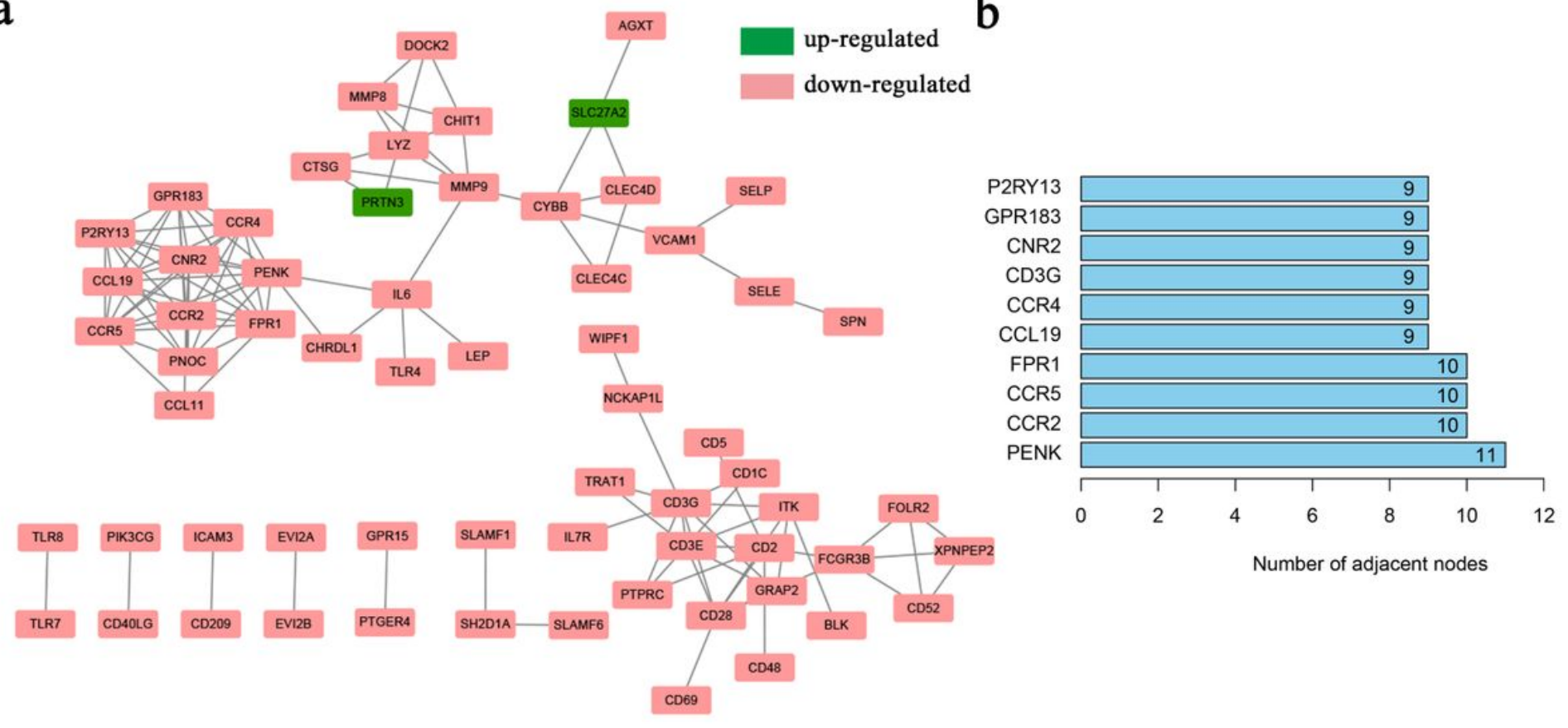

c

\begin{tabular}{|c|c|c|}
\hline & e & \\
\hline JAML & 0.014 & $0.820(0.700-0.961)$ \\
\hline SIT1 & 0.031 & $0.925(0.861-0.993)$ \\
\hline CAMK4 & 0.041 & $0.544(0.303-0.974)$ \\
\hline ITK & 0.040 & $0.834(0.701-0.992)$ \\
\hline CD48 & 0.015 & $0.958(0.925-0.992)$ \\
\hline $\mathrm{CD} 2$ & 0.021 & $0.982(0.967-0.997)$ \\
\hline TESPA1 & 0.023 & $0.762(0.602-0.963)$ \\
\hline CD226 & 0.031 & $0.454(0.222-0.929)$ \\
\hline CLEC10A & 0.032 & $0.922(0.856-0.993)$ \\
\hline SCML4 & 0.045 & $0.543(0.299-0.988)$ \\
\hline KLRB1 & $<0.001$ & $0.816(0.725-0.917)$ \\
\hline IL $12 B$ & 0.011 & $0.230(0.074-0.718)$ \\
\hline CD5 & 0.027 & $0.937(0.885-0.993)$ \\
\hline CD4OLG & 0.026 & $0.823(0.693-0.977)$ \\
\hline CCL19 & 0.014 & $0.994(0.990-0.999)$ \\
\hline SLAMF6 & 0.035 & $0.936(0.881-0.995)$ \\
\hline UBASH 3 A & 0.019 & $0.765(0.612-0.957)$ \\
\hline GZMM & 0.019 & $0.907(0.835-0.984)$ \\
\hline CRTAM & 0.033 & $0.743(0.566-0.976)$ \\
\hline c1s & 0.039 & $0.996(0.992-1.000)$ \\
\hline CD3E & 0.012 & $0.971(0.948-0.993)$ \\
\hline XCR1 & 0.047 & $0.594(0.355-0.993)$ \\
\hline GPR171 & 0.011 & $0.817(0.698-0.956)$ \\
\hline TRAT1 & 0.047 & $0.829(0.688-0.998)$ \\
\hline CLEC9A & 0.017 & $0.358(0.154-0.832)$ \\
\hline CD1E & 0.029 & $0.872(0.771-0.9$ \\
\hline IL7F & 0.036 & $0.968(0.940-0$. \\
\hline $\mathrm{CD} 2$ & 0.016 & $0.953(0.917-0$ \\
\hline CD52 & 0.004 & $0.988(0.980-c$ \\
\hline & 0.026 & $0.893(0.808-$ \\
\hline & 0.030 & $.901(0.819-$ \\
\hline CST7 & 0.004 & $0.958(0.931-0$ \\
\hline
\end{tabular}

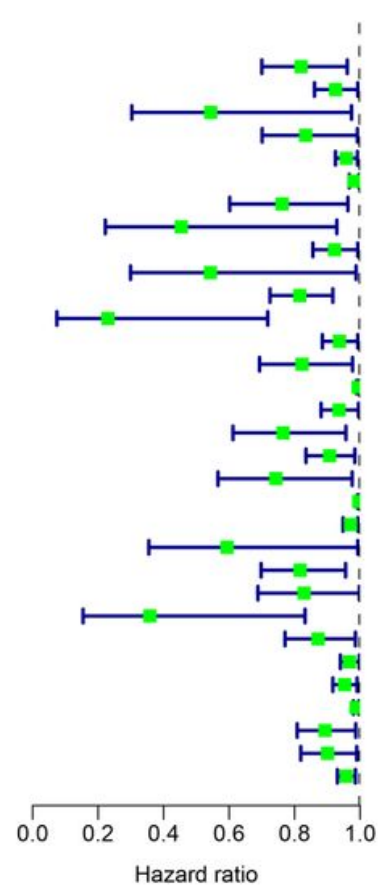

d

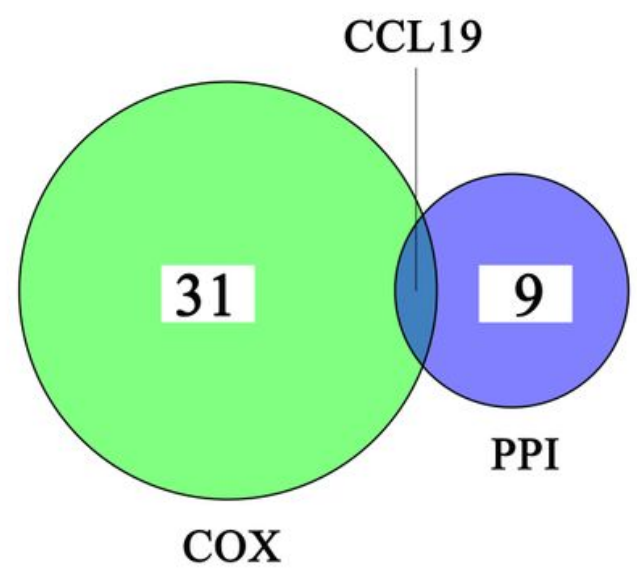

Figure 4

PPI network construction and univariate COX regression analysis. (a) PPI network was constructed with interaction confidence value $>0.95$; (b) The top 10 DEGs which shared the leading nodes in PPI network; (c) Univariate COX regression analysis of 223 DEGs. $p<0.05$ was considered to be statistically significant; (d) Venn plot displayed the DEGs which shared the top 10 leading nodes in PPI network and were significant related with patients' survival. $p<0.05$ was considered to be statistically significant. 


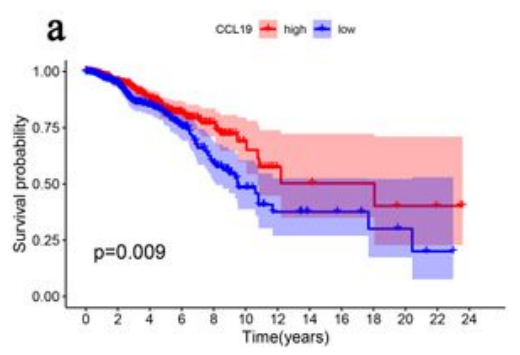

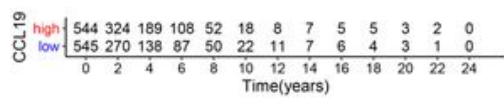

d

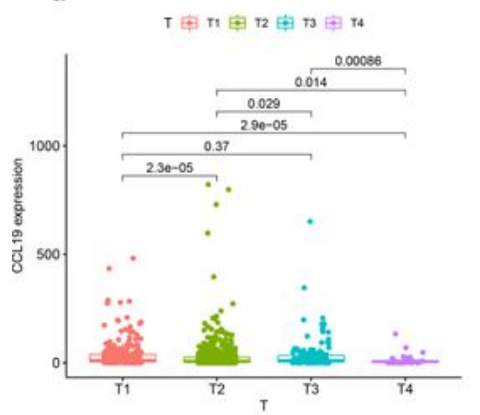

g
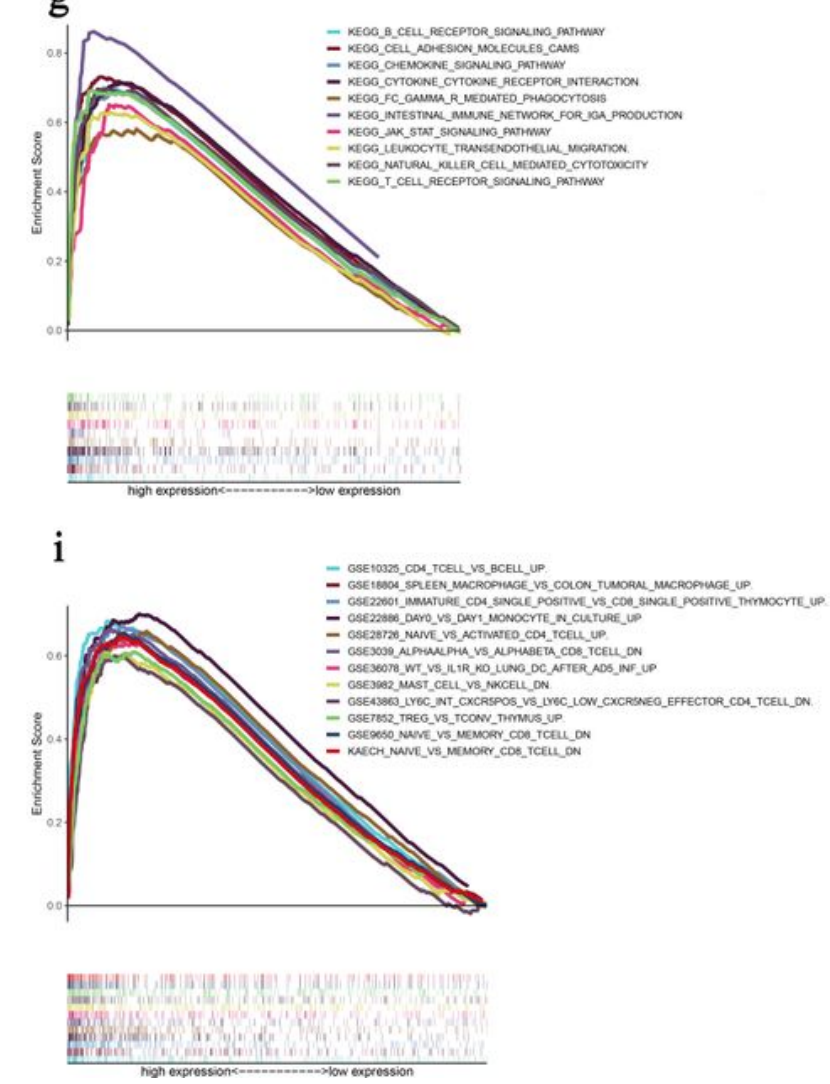

b

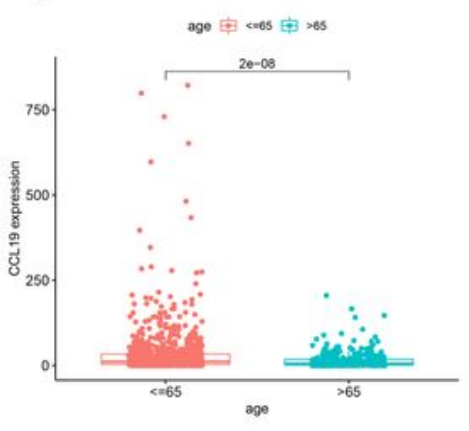

e

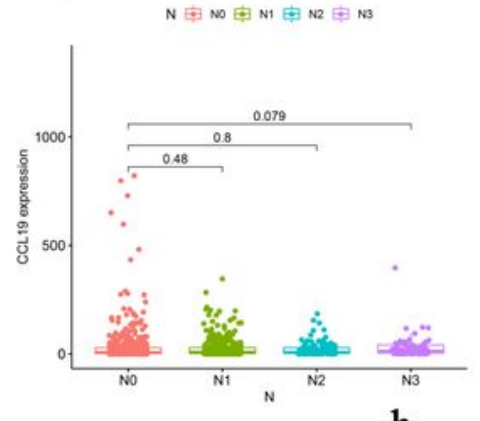

c

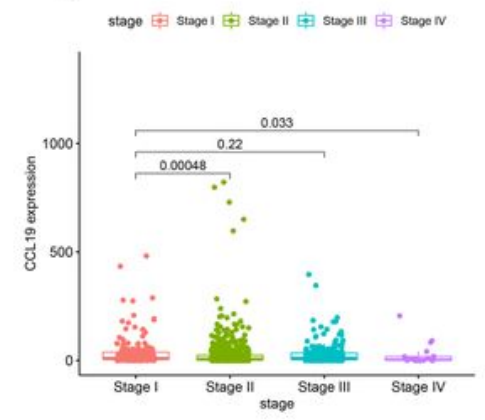

f

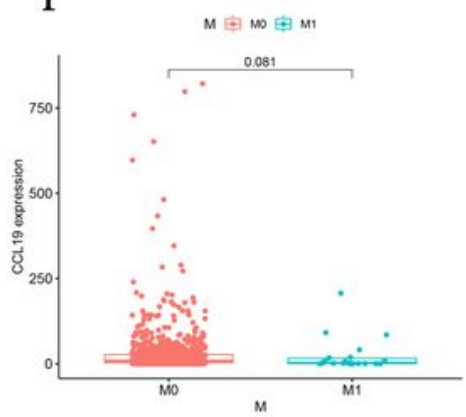

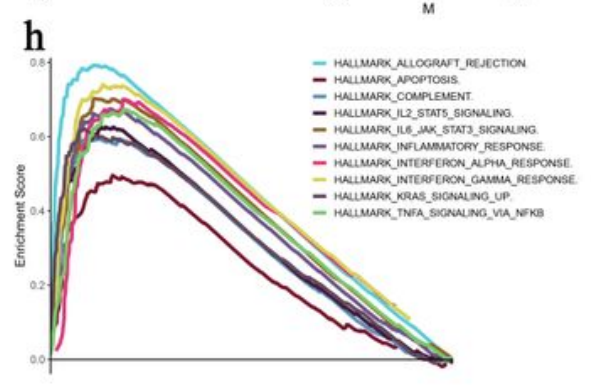
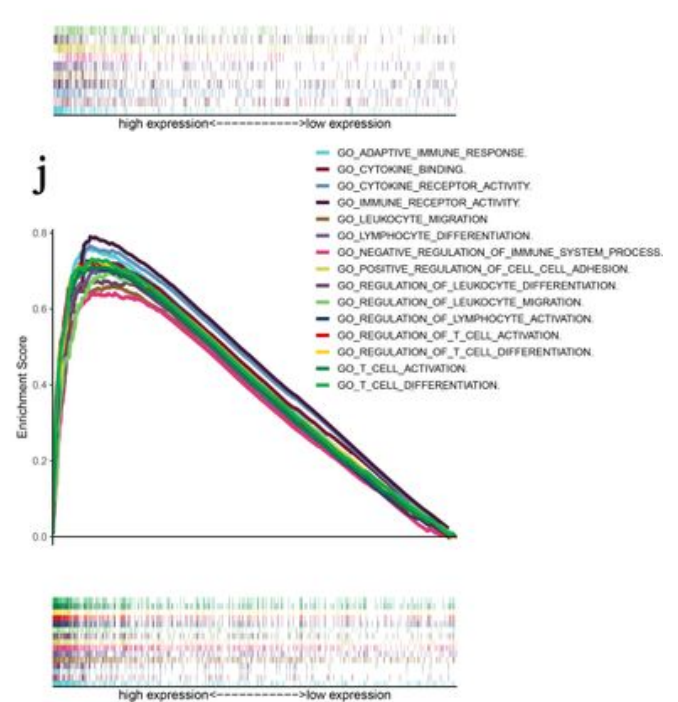

\section{Figure 5}

The survival analysis, differential analysis and GSEA of CCL19. (a) The survival analysis between the expression of CCL19 and the survival of breast cancer patients; patients were divided into two groups, high CCL19 expression group and low CCL19 expression group, compared with the median; (b-f) The associations between the expression of CCL19 with age, stage, and TNM classification of breast cancer 
patients; $p$ value was displayed in the diagram; (g-j) GSEA for genes in high CCL19 expression group in different gene sets, $p<0.05$ and FDR $<0.05$ were considered to be statistically significant.

a

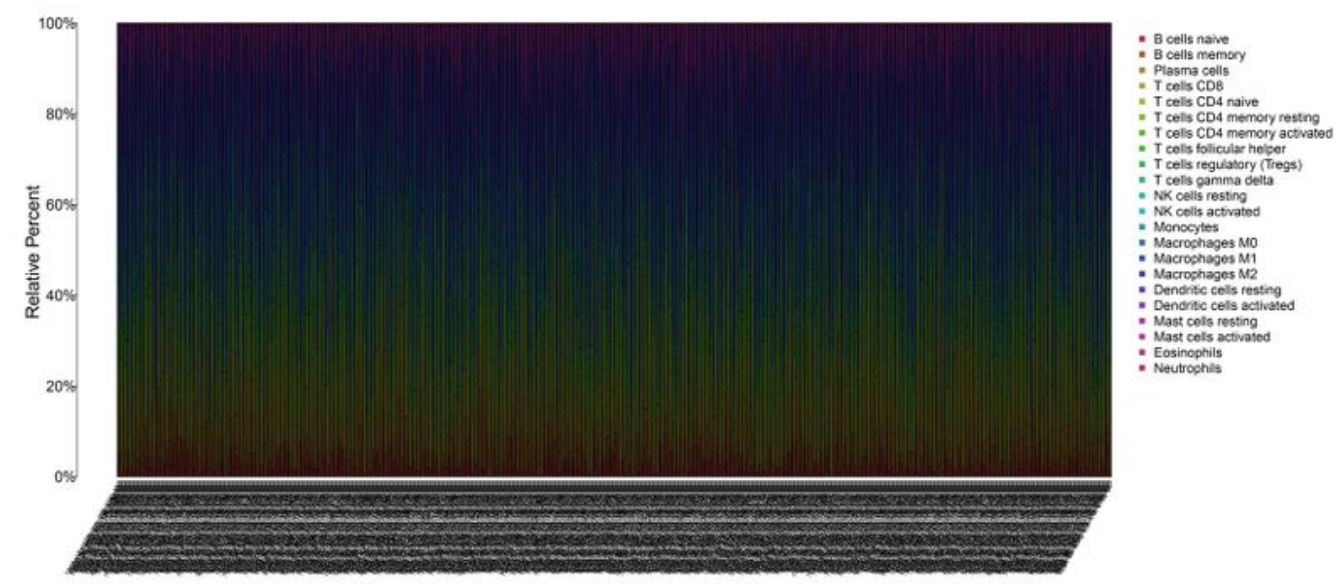

b

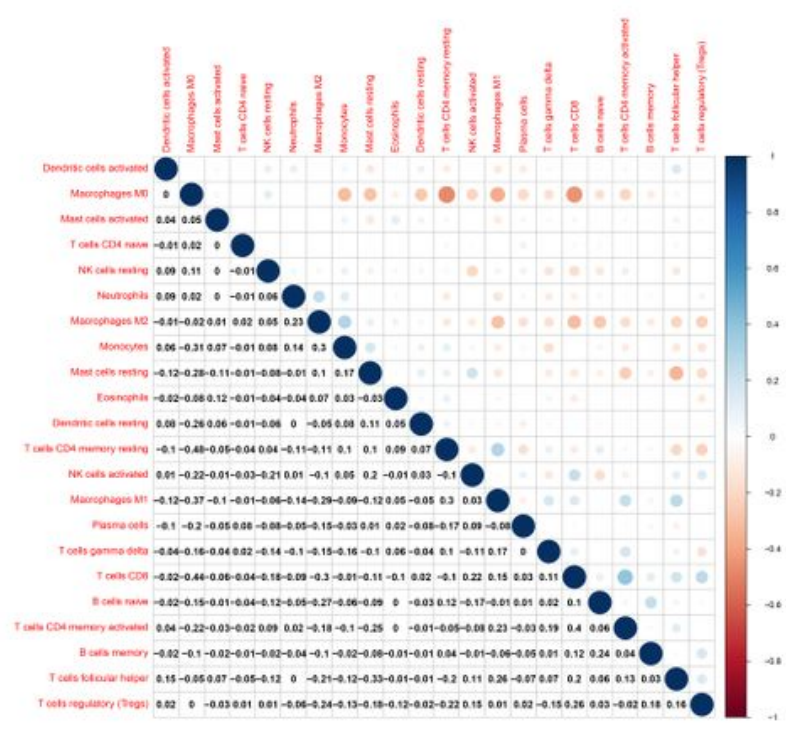

c

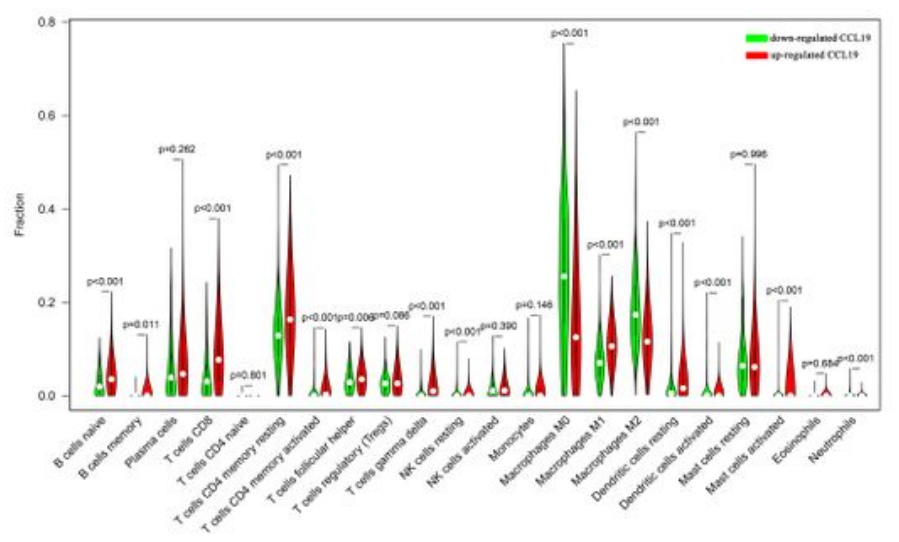

d

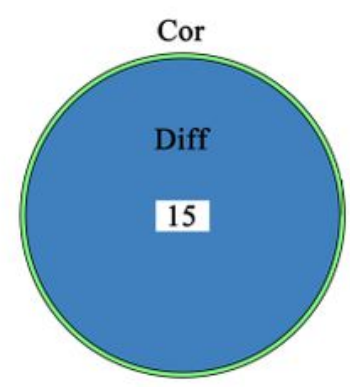

Figure 6

TIC profile and differential analysis. (a) Barplot showed the proportion of 22 TICs in breast cancer samples; (b) The diagram showed the associations between 22 TICs; each spot indicated the $p$ value of the association between two TICs; (c) Violin plot showed the correlation between 22 TICs and the 
expression of CCL19; $p<0.05$ was considered to be statistically significant; (d) Venn plot indicated there were 15 TICs shared by the difference test and correlation test showed in violin plots (c) and scatter plots (Figure 7), respectively.
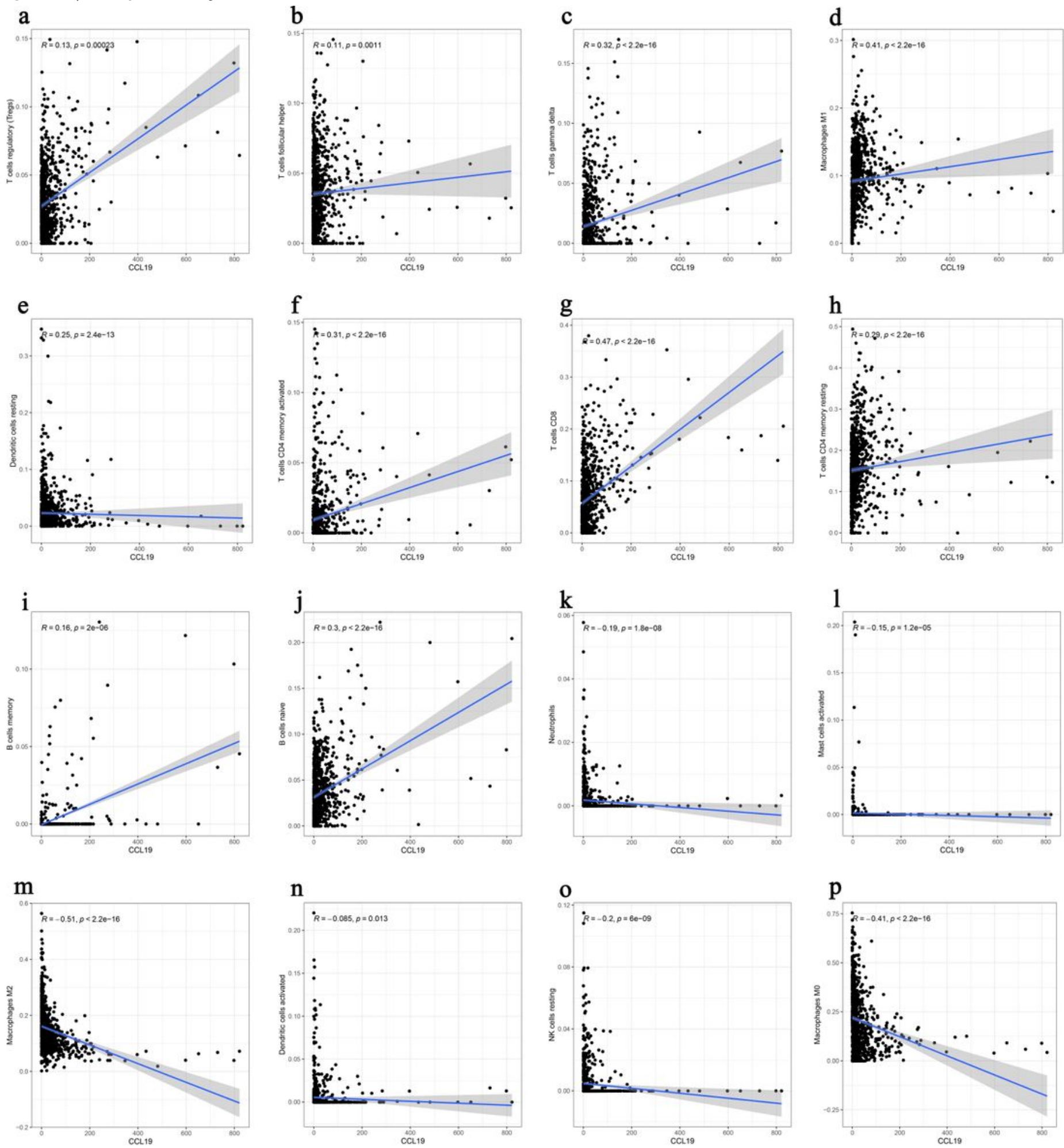

Figure 7

Scatter plots indicated the correlation between 16 TICs and the expression of CCL19, $p<0.05$ was considered to be statistically significant. 


\section{Supplementary Files}

This is a list of supplementary files associated with this preprint. Click to download.

- SupplementTable1.docx

- SupplementTable2.docx

- SupplementTable3.docx

- SupplementTable4.docx

- SupplementTable5.docx

- SupplementTable6.docx 